\title{
Enhanced expression of hydroxylated ceramide in well-differentiated endometrial adenocarcinoma
}

\author{
TOSHIKI TAJIMA ${ }^{1}$, MASAKI MIYAZAWA ${ }^{1}$, MASARU HAYASHI $^{1}$, SATOSHI ASAI $^{1}$, MASAE IKEDA $^{1}$, \\ MASAKO SHIDA ${ }^{1}$, TAKESHI HIRASAWA ${ }^{1}$, MASAO IWAMORI $^{2}$ and MIKIO MIKAMI ${ }^{1}$ \\ ${ }^{1}$ Department of Obstetrics and Gynecology, Tokai University School of Medicine, Isehara, Kanagawa 259-1193; \\ ${ }^{2}$ Department of Biochemistry, Faculty of Science and Technology, Kinki University, Higashiosaka, Osaka 577-8502, Japan
}

Received April 6, 2015; Accepted June 27, 2016

DOI: $10.3892 / 01.2016 .5410$

\begin{abstract}
Based on our previous analysis of neutral glycolipids in the human endometrium, the present authors already reported that the concentrations of glucosylceramide, lactosylceramide and globotriaosylceramide $\left(\mathrm{Gb}_{3} \mathrm{Cer}\right)$, in which both fatty acids and sphingosines in the ceramides are hydroxylated, exhibit a marked increase during the luteal phase of the menstrual cycle. It is also well known that poorly differentiated endometrial adenocarcinoma exhibits a more rapid progression and a worse response to therapy than well-differentiated endometrial adenocarcinoma. To examine the molecular background of well-differentiated and poorly differentiated cancers, the levels of neutral glycolipids in tumor tissues from endometrial carcinoma displaying different degrees of differentiation were measured. The composition of neutral glycolipids in tumor tissues was determined, and ceramide structures that were specifically expressed in well-differentiated endometrial carcinomas were investigated using biochemical analytical methods, including lipid extraction, enzyme digestion, thin-layer chromatography (TLC), gas-liquid chromatography and mass spectrometry. Well-differentiated adenocarcinoma contained numerous structurally unknown glycolipids that exhibited slower migration than globotetraosylceramide $\left(\mathrm{Gb}_{4} \mathrm{Cer}\right)$. In the case of $\mathrm{Gb}_{3} \mathrm{Cer}$, three bands appeared on TLC in well-differentiated cancer, but only two bands appeared in the poorly-differentiated cancer. This difference was associated with the fatty acid composition of ceramide, since non-hydroxy fatty acids with $\geq 20$ carbon atoms were increased in well-differentiated cancer, while $\alpha$-hydroxy fatty acids were increased in poorly
\end{abstract}

Correspondence to: Dr Mikio Mikami, Department of Obstetrics and Gynecology, Tokai University School of Medicine, 143 Shimokasuya, Isehara, Kanagawa 259-1193, Japan

E-mail: mmikami@is.icc.u-tokai.ac.jp

Abbreviations: TLC, thin-layer chromatography; MS, mass spectrometry

Key words: phytosphingosine, glycolipid, endometrial cancer, differentiation, $\alpha$-hydroxy fatty acid differentiated cancer. Similarly, there were two bands on TLC of $\mathrm{Gb}_{4}$ Cer from well-differentiated cancer, but only one band in poorly differentiated cancer, and the long-chain base of ceramide was observed to contain phytosphingosine in well-differentiated cancer. It was demonstrated in endometrial cancer that the structure of ceramide molecules changes with the extent of tumor differentiation. These findings suggest that hydroxylated ceramides contribute to the well-differentiated phenotype of endometrial adenocarcinoma.

\section{Introduction}

Since glycolipids exhibit a tissue- and cell-specific distribution, and their composition changes markedly during processes such as carcinogenesis, differentiation and proliferation, these molecules are considered to be important for cellular functions (1). The number of reports concerning the physiological role of glycolipids has increased in the past years, and attention has been focused on their functions (1). The present authors have analyzed glycolipids in the human endometrium, where differentiation and proliferation are controlled by sex hormones, and have already reported that there is a marked increase of sulfatides with sulfate groups in the secretory endometrium (2), and that the concentrations of glucosylceramide (GlcCer), lactosylceramide (LacCer) and globotriaosylceramide $\left(\mathrm{Gb}_{3} \mathrm{Cer}\right)$ (which contain a ceramide in which the fatty acids and sphingosine are hydroxylated) exhibited a marked increase during the secretory phase of the menstrual cycle (3). In a previous study on breast cancer (4), it was also reported that ceramide structures with specific chain lengths are very different in estrogen receptor (ER)-positive tumors compared with ER-negative tumors. Based on these observations, it could be proposed that the ceramide expressed in cancer cells may have specific functions influenced by sex steroid hormones. Ceramide is synthesized from fatty acids and a long-chain base, with the fatty acids being classified as $\alpha$-hydroxy fatty acids if they have a hydroxyl group, or non-hydroxy fatty acids if they lack such a group (5). The long-chain base is mostly composed of sphingosine, but it can be synthesized from phytosphingosine (in which a hydroxyl group is attached to sphingosine) or from dihydrosphingosine (in which the double bond at position 4 of sphingosine is saturated) (6). 
An association has been reported between the prognosis of endometrial carcinoma and tumor differentiation (gland formation) (7). Poorly differentiated endometrial adenocarcinoma exhibits more rapid progression and is less responsive to therapy than well-differentiated adenocarcinoma, resulting in a worse prognosis (8). Diagnosis of tumor differentiation is usually performed by pathologists, and the tumor grade assigned may be the single most important prognostic factor for endometrial cancer (9). However, little basic research has been conducted on the process of transformation of endometrial cancer to the well-differentiated or poorly differentiated phenotype. Understanding the mechanism involved in the differentiation of endometrial cancer may lead to novel therapies that induce tumor differentiation.

Accordingly, the objectives of the present study were to compare the expression of neutral glycolipids between well-differentiated and poorly differentiated adenocarcinoma of the endometrium, focusing on the ceramide moiety, and to investigate the role of glycolipids in morphological differentiation (gland formation), which is the most important prognostic factor for endometrial cancer.

\section{Materials and methods}

Materials. Glycolipids from various sources were purified at the Department of Obstetrics and Gynecology, Tokai University (Tokai University School of Medicine, Isehara, Japan), including GlcCer, $\mathrm{LacCer}, \mathrm{Gb}_{3} \mathrm{Cer}$ and globotetraosylceramide $\left(\mathrm{Gb}_{4} \mathrm{Cer}\right)$, from human erythrocytes, which were obtained from the Japanese Red Cross (Tokyo, Japan).

Tumor tissues. Tumor tissues were obtained from the Department of Obstetrics and Gynecology at Tokai University Hospital (Isehara, Japan). Written informed consent for the use of tumor specimens in the present study was obtained from all subjects, and the experimental protocol was approved by the Ethics Committee of Tokai University Hospital (Institutional Review Board approval no. 09R-097). The histological classification of the tumors was performed according to the criteria of the International Federation of Gynecology and Obstetrics (10). Tumor differentiation was diagnosed histologically according to the amount of glandular and solid areas in the cancerous tissue. A total of 24 endometrial adenocarcinomas were studied, and 9 well-differentiated tumors, 11 moderately differentiated tumors and 4 poorly differentiated tumors were used for biochemical analysis. Tumor samples were immediately stored at $-70^{\circ} \mathrm{C}$ until use. For histological examination, tissues were fixed in formalin and embedded in paraffin, and subsequently, sections (4- $\mu$ m-thick) were cut and stained with hematoxylin and eosin.

Quantitative determination of neutral glycolipids from tumor tissues. Tumor tissues were homogenized by Polytron homogenizer (Kinematica, Luzern, Switzerland) with water and were lyophilized. Total lipids were extracted from the lyophilized powder with chloroform/methanol/water (20:10:1, 10:20:1 and 1:1, v/v). Then, the lipid extracts were fractionated into neutral and acidic lipids on a DEAE Sephadex ${ }^{\mathrm{TM}}$ A-25 column in acetate form (Pharmacia Biotech; GE Healthcare Life Sciences, Uppsala, Sweden). Preparation of neutral glycolipids was performed as described previously (11-13). The neutral glycolipids were separated from the unabsorbed neutral lipid fraction by acetylation, separation of the acetylated derivatives, deacetylation and desalting. The neutral glycolipids obtained were developed on thin-layer chromatography (TLC) plates (Merck, Darmstadt, Germany) with chloroform/methanol/water $(65: 35: 8, \mathrm{v} / \mathrm{v} / \mathrm{v})$, and then visualized with orcinol- $\mathrm{H}_{2} \mathrm{SO}_{4}$ (Wako Pure Chemical Industries, Tokyp, Japan). The density of the spots was determined at an analytical wavelength of $420 \mathrm{~nm}$ for orcinol- $\mathrm{H}_{2} \mathrm{SO}_{4}$-positive spots, using a dual-wavelength TLC densitometer (CS-9000; Shimadzu Corporation, Kyoto, Japan). Standard glycolipids (Funakoshi Co., Ltd., Tokyo, Japan), which were N-stearoyl derivatives of GlcCer, LacCer and $\mathrm{Gb}_{3} \operatorname{Cer}(0.1-1.5 \mu \mathrm{g})$, were developed on the same TLC plates to generate standard curves for quantitation.

Isolation and structural analysis of specific neutral glycolipids Isolation. Only 1 well-differentiated case (lane 1 in Fig. 1) and 1 poorly-differentiated case (lane 17 in Fig. 1) were selected for analysis. The neutral glycolipids from well-differentiated and poorly differentiated adenocarcinoma were purified on a column (1.8-cm inner diameter and 55.0-cm length) packed with Iatrobeads ${ }^{\circledR}$ (Iatron Lab. Inc., Tokyo, Japan), with a linear gradient system formed from chloroform/methano1/water (85:15:0.5 and 20:80:5, v/v/v) (3).

Under these conditions, individual bands corresponding to $\mathrm{Gb}_{3} \mathrm{Cer}$ and $\mathrm{Gb}_{4} \mathrm{Cer}$ specifically expressed in both adenocarcinomas were successfully isolated in a pure form. The homogeneity of the isolated neutral glycolipids was examined by TLC with orcinol- $\mathrm{H}_{2} \mathrm{SO}_{4}$ reagent.

Fatty acid and long-chain base compositions of ceramides in neutral glycolipids specifically expressed in endometrial adenocarcinoma. The isolated glycolipids were treated with $0.75 \mathrm{M}$ methanolic $\mathrm{HCl}$ at $80^{\circ} \mathrm{C}$ for $20 \mathrm{~h} \mathrm{(4),} \mathrm{and} \mathrm{then} \mathrm{the}$ fatty acid methyl esters were extracted from the hydrolyzates with n-hexane. The samples were analyzed by gas-liquid chromatography using 3\% OV-101 (GL Science, Inc., Tokyo, Japan) on ChroLite (100-120 mesh; Shimadzu Corporation), with a programmed temperature increase of $1^{\circ} \mathrm{C} / \mathrm{min}$ from $150-250^{\circ} \mathrm{C}$, and were characterized with non-hydroxy fatty acids and $\alpha$-hydroxy fatty acids (Wako Pure Chemical Industries). The peak areas obtained were corrected by comparison with the peak areas of an authentic mixture of fatty acid methyl esters (Applied Science Labs., State College, PA, USA). The long-chain bases were also extracted from the hydrolyzates with diethyl ether after changing the $\mathrm{pH}$ to 11 with $1 \mathrm{M} \mathrm{NaOH}$, and were developed on a TLC plate with sphingosine, dihydrosphingosine and phytosphingosine (Sigma-Aldrich, St. Louis, MO, USA) using chloroform/methanol/2 N ammonia (40:10:1, $\mathrm{v} / \mathrm{v})(11)$, and visualized with ninhydrin reagent.

Structural analysis of specific glycolipids. The structures of the further purified glycolipids were also determined by negative ion fast atom bombardment mass spectrometry (FABMS) and glycosidase treatment. Approximately $5 \mu \mathrm{g}$ of an isolated neutral glycolipid in $5 \mu \mathrm{l}$ of chloroform/methanol $(1: 1, \mathrm{v} / \mathrm{v})$ was mixed with $\sim 5 \mu \mathrm{l}$ of triethanolamine, and the resultant solution was placed on a stainless steel sample holder for FABMS. 


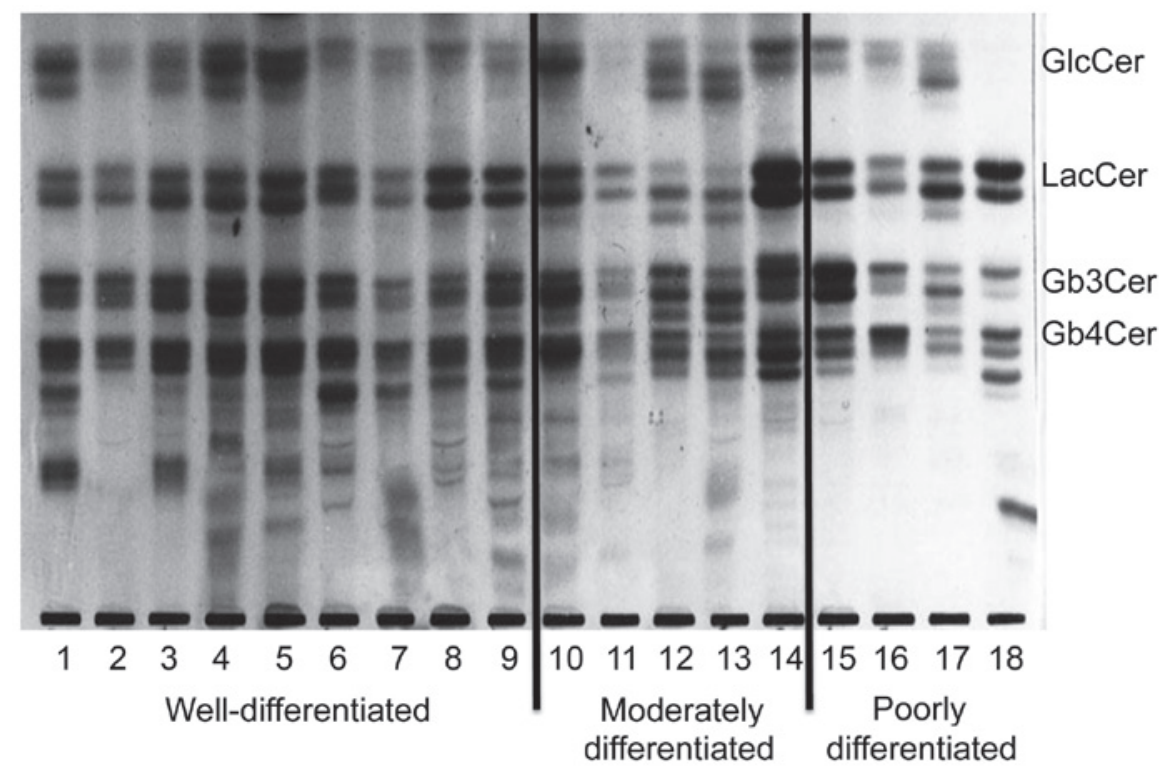

Figure 1. Thin-layer chromatography of neutral glycolipids from human endometrial adenocarcinoma of various degrees of differentiation. The neutral glycolipids obtained from cancerous tissues (lanes 1-18) were chromatographed with chloroform/methanol/water (65:35:8, v/v) and located by spraying orcinol- $\mathrm{H}_{2} \mathrm{SO}_{4}$ reagent and heating at $110^{\circ} \mathrm{C}$ for $10 \mathrm{~min}$. Lanes 1-9 are well-differentiated adenocarcinoma tissues, while lanes 10-14 and 15-18 correspond to moderately and poorly differentiated adenocarcinoma tissues, respectively. GlcCer, glucosylceramide; LacCer, lactosylceramide; $\mathrm{Gb}_{3} \mathrm{Cer}$, globotriaosylceramide; $\mathrm{Gb}_{4} \mathrm{Cer}$, globotetraosylceramide.

A

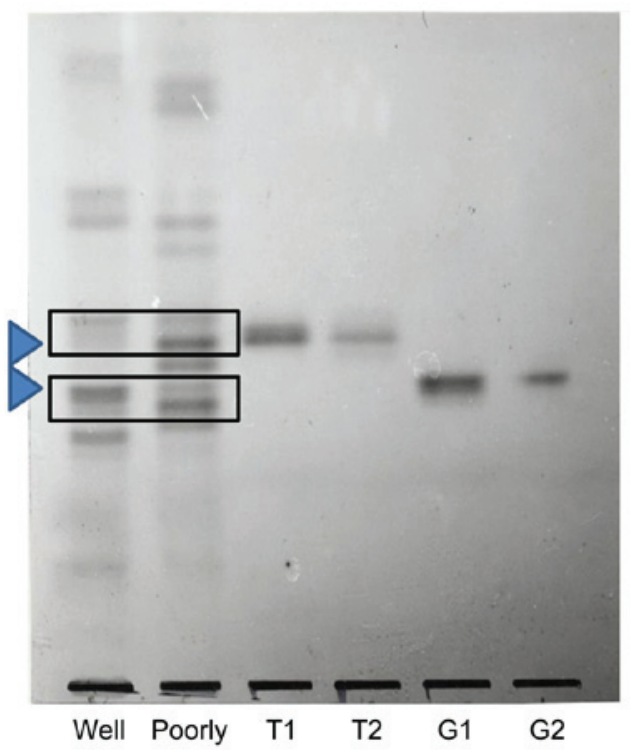

B

GlcCer

LacCer

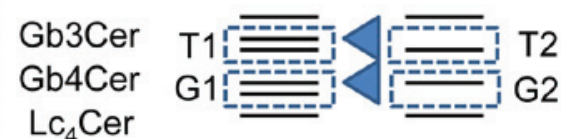

Well Poorly

Figure 2. (A) TLC of the constituents of the $\mathrm{Gb}_{3} \mathrm{Cer}$ and $\mathrm{Gb}_{4} \mathrm{Cer}$ bands isolated from well- (bands $\mathrm{T} 1$ and G1) and poorly (bands $\mathrm{T} 2$ and G2) differentiated endometrial adenocarcinoma. Neutral glycolipids were isolated from the total neutral lipid fraction as described in Materials and methods. The total neutral glycolipids and the isolated glycolipids were developed with chloroform/methanol/water (65:35:8, v/v) and visualized with orcinol- $\mathrm{H}_{2} \mathrm{SO}_{4}$ reagent. Lanes Well and Poorly correspond to the total neutral glycolipids in well- and poorly differentiated adenocarcinoma, respectively. Lanes T1, T2, G1 and G2 are the isolated neutral glycolipids from the three bands of $\mathrm{Gb}_{3} \mathrm{Cer}$ and the band of $\mathrm{Gb}_{4} \mathrm{Cer}$, respectively. (B) TLC schema of T1, T2, G1 and G2. TLC, thin-layer chromatography; GlcCer, glucosylceramide; LacCer, lactosylceramide; $\mathrm{Gb}_{3} \mathrm{Cer}$, globotriaosylceramide; $\mathrm{Gb}_{4} \mathrm{Cer}$, globotetraosylceramide; $\mathrm{Lc}_{4} \mathrm{Cer}$, lactotetraosyl ceramide .

Analysis was performed by bombardment with a neutral xenon beam with a kinetic energy of $4 \mathrm{keV}$, and detection of negative ions was performed with a mass spectrometer (JMSHX-110; JEOL, Ltd., Tokyo, Japan) equipped with a JMA-5500 computer system (JEOL,Ltd.). Assignment of mass numbers was achieved by comparing the spectrum with that of perfluoroalkyl phosphazine (Ultramark 1621; PCR, Inc., Gainesville, FL, USA).

TLC upon treatment with $\alpha$-galactosidase (Sigma-Aldrich) was performed to confirm the sequence of the sugar chains.

\section{Results}

Expression of neutral glycolipids in well-, moderately and poorly differentiated adenocarcinoma of the endometrium. GlcCer, LacCer, $\mathrm{Gb}_{3} \mathrm{Cer}$ and $\mathrm{Gb}_{4} \mathrm{Cer}$ were the principal neutral glycolipids identified in endometrial cancer (Fig. 1). In well-differentiated tumors, numerous structurally unknown glycolipids exhibiting slower migration than $\mathrm{Gb}_{4} \mathrm{Cer}$ were also identified, although there was individual variation. $\mathrm{Gb}_{3} \mathrm{Cer}$ 


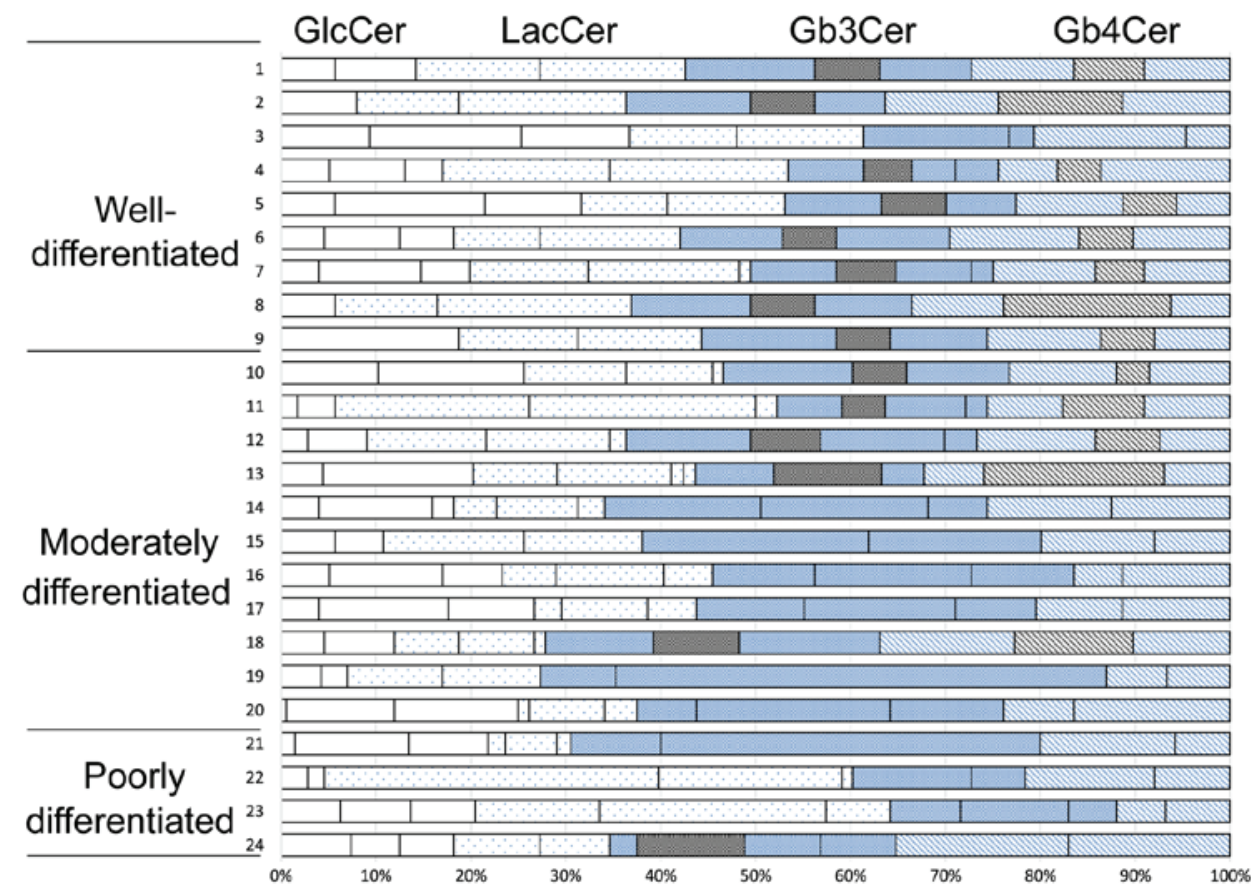

Figure 3. Relative expression of each band of glucosylceramide, lactosylceramide, globotriaosylceramide and globotetraosylceramide in well-, moderately and poorly differentiated adenocarcinoma of the endometrium. Bands containing phytosphingosine in $\mathrm{Gb}_{3} \mathrm{Cer}_{\text {and }} \mathrm{Gb}_{4} \mathrm{Cer}$ of well differentiated adenocarcinoma are designated as black closed column. The relative amount of this characteristic band was shown. GlcCer, glucosylceramide; LacCer, lactosylceramide; $\mathrm{Gb}_{3} \mathrm{Cer}$, globotriaosylceramide; $\mathrm{Gb}_{4} \mathrm{Cer}$, globotetraosylceramide.

A

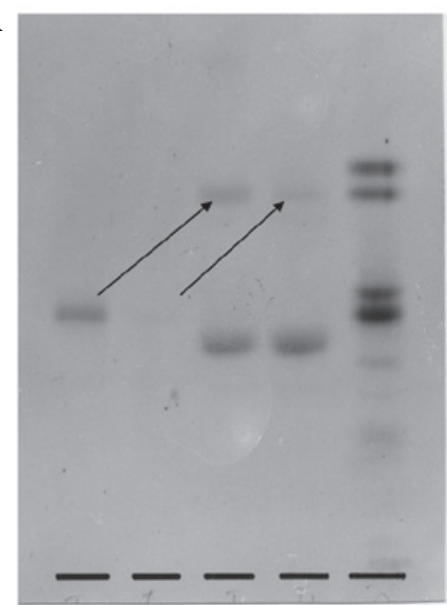

T1

After enzyme treatment
B

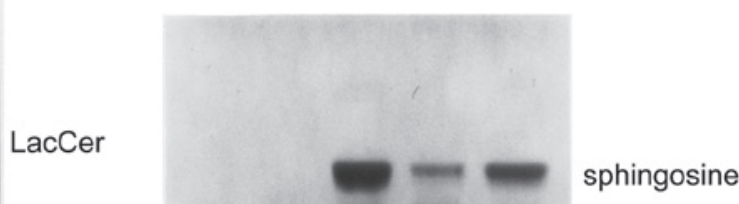

Gb3Cer

taurocholate

E dihydrosphingosine

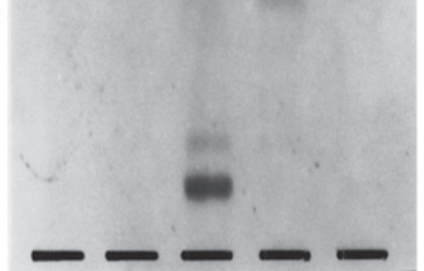

T1 $\quad$ T2 $\quad$ G1 1 G2 standard

phytosphingosine

Figure 4. (A) Structural analysis of the sugar chains of LacCer, as indicated by arrows, by TLC following enzyme treatment with $\alpha$-galactosidase. Upon treatment with the enzyme, both T1 and T2 changed to LacCer, and both were observed to be $\mathrm{Gb}_{3} \mathrm{Cer}$ with a terminal $\alpha$-galactose. (B) Analysis of the composition of the long-chain base by TLC. Dihydrosphingosine was noticed to be the principal component in both T1 and T2, while sphingosine or phytosphingosine were not detected in either of them. However, TLC analysis revealed that the ceramide of G1 was composed of sphingosine, dihydrosphingosine and phytosphingosine, while G2 contained just sphingosine and dihydrosphingosine. TLC, thin-layer chromatography; LacCer, lactosylceramide; $\mathrm{Gb}_{3} \mathrm{Cer}_{\text {, }}$ globotriaosylceramide.

(indicated by the top arrow in Fig. 2A and B) had two components in well-differentiated cancer, while it had only one component in poorly differentiated cancer. $\mathrm{Gb}_{4} \mathrm{Cer}$ (indicated by the bottom arrow in Fig. 2A and B) was also composed of two bands in well-differentiated cancer, while it was composed of only one band in poorly differentiated cancer. Thus, a band that was not noted in poorly differentiated cancer was observed to be present in $\mathrm{Gb}_{3} \mathrm{Cer}$ and $\mathrm{Gb}_{4} \mathrm{Cer}$ from well-differentiated cancer. Fig. 3 represents the relative amount of these characteristic bands. These observations suggest that specific bands of $\mathrm{Gb}_{3} \mathrm{Cer}$ and $\mathrm{Gb}_{4} \mathrm{Cer}$ were characteristically more expressed in well-differentiated adenocarcinoma than in poorly differentiated adenocarcinoma.

Structural analysis. The $\mathrm{Gb}_{3} \mathrm{Cer}$ and $\mathrm{Gb}_{4} \mathrm{Cer}$ bands exhibited differences between well- and poorly differentiated cancer, and 

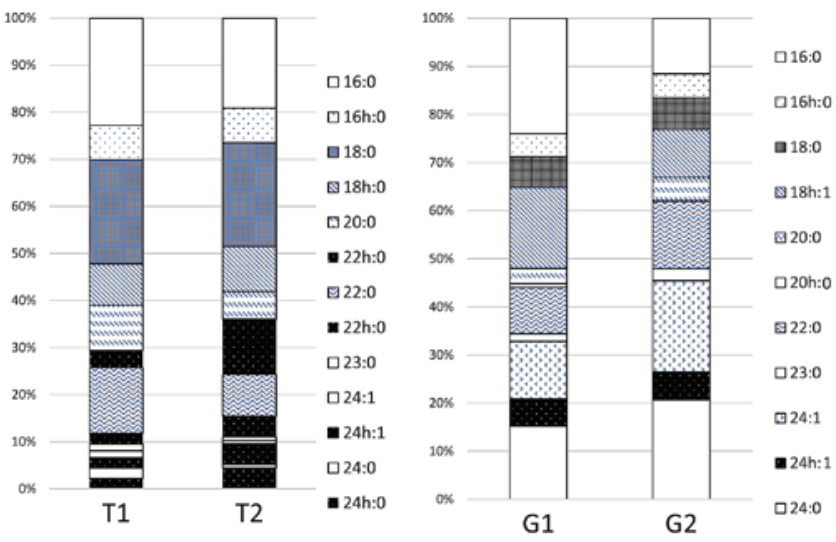

Figure 5. Relative fatty acid compositions of T1, T2, G1 and G2. Each fatty acid is designated as the number of carbon atoms : number of double bonds that contains. There were no appreciable differences between T1 and $\mathrm{T} 2$ when the number of carbon atoms was 16 or 18 . However, when it was $\geq 20$, the proportion of non-hydroxy fatty acids without hydroxyl groups was higher in T1 than in T2, while $\alpha$-hydroxy fatty acids were predominant in T2. No appreciable differences between G1 and G2 were observed. h, hydroxy fatty acids.

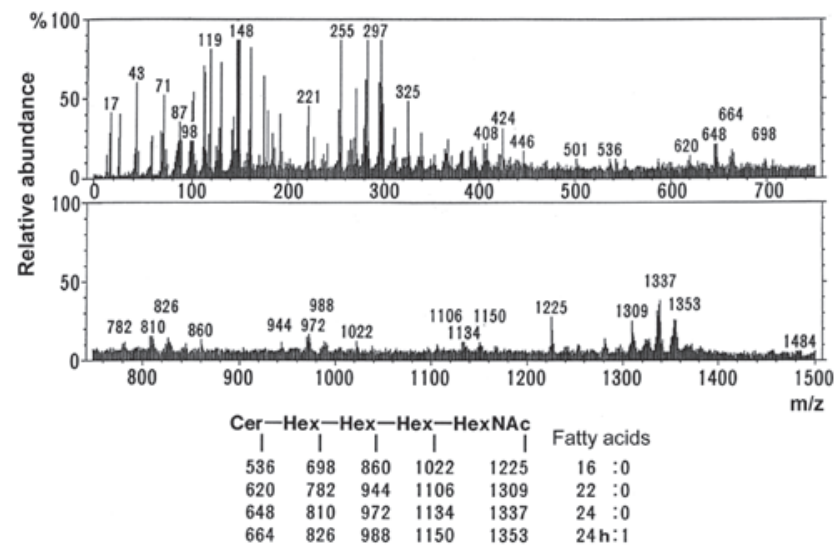

Figure 6. Negative ion fast atom bombardment mass spectrometry spectra of G2 that correspond to glycolipids in Fig. 2A, from poorly differentiated adenocarcinoma. Approximately $5 \mu \mathrm{g}$ each of the glycolipids in $\mathrm{G} 2$ were mixed in triethanolamine and then analyzed with a mass spectrometer (JMSHX-110; JEOL, Ltd.). The molecular ion peaks shown in the lower part of Fig. 6 indicated that the structure was Cer-Hex-Hex-Hex-HexNAc (being Cer, ceramide; Hex, hexose; and HexNAc, N-acetylhexoseamine). These results suggested that the sugar chains of the G1 and G2 bands were globotetraosylceramide. h, hydroxy fatty acids.

were isolated and purified using a Iatrobeads column (Fig. 2A and $\mathrm{B}$ ). The $\mathrm{Gb}_{3}$ Cer bands with a lower rate of migration on TLC isolated from well- and poorly differentiated cancer were designated as $\mathrm{T} 1$ and $\mathrm{T} 2$, respectively, while the $\mathrm{Gb}_{4}$ Cer bands isolated from well- and poorly differentiated cancer were called G1 and G2, respectively. Differences between T1 and T2 or between G1 and G2 were investigated with respect to sugar chains, fatty acids and the long-chain base as constituents of ceramide.

$\alpha$-Galactosidase was reacted with $\mathrm{T} 1$ and $\mathrm{T} 2$ prior to structural analysis by TLC (Fig. 4A). Following treatment with the enzyme, both T1 and T2 changed to LacCer. Both were noticed to be $\mathrm{Gb}_{3} \mathrm{Cer}$ with a terminal $\alpha$-galactose, and the sugar chain of the band in $\mathrm{T} 1$ that was not observed in poorly differentiated cancer was also identified as $\mathrm{Gb}_{3}$ Cer. Fig. 5 displays the results of the analysis of the fatty acid composition of ceramide. There were no appreciable differences between $\mathrm{T} 1$ and T2 when the number of carbon atoms was 16 or 18 . When the number of carbon atoms was $\geq 20$, however, the proportion of non-hydroxy fatty acids without hydroxyl groups was higher in $\mathrm{T} 1$ than in T2, while $\alpha$-hydroxy fatty acids were predominant in T2. Fig. 4B displays the results of the analysis of the composition of the long-chain base by TLC. Dihydrosphingosine was observed to be the principal component in both $\mathrm{T} 1$ and T2, while sphingosine or phytosphingosine were not detected in either of them. These results suggested that the band detected in $\mathrm{T} 1$ but not in $\mathrm{T} 2$ was due to differences on the fatty acid composition.

As shown in Fig. 2A and B, the migration of the upper band in G1 and G2 on TLC corresponded to the migration of $\mathrm{Gb}_{4} \mathrm{Cer}$, while the band from G1 was located midway between $\mathrm{Gb}_{4} \mathrm{Cer}$ and lactotetraosyl ceramide ( $\left.\mathrm{Lc}_{4} \mathrm{Cer}\right)$. Therefore, this band from G1 was likely to be $\mathrm{Lc}_{4} \mathrm{Cer}$ or neolactotetraosyl ceramide $\left(\mathrm{nLc}_{4} \mathrm{Cer}\right)$. However, TLC immunostaining using anti-type I and anti-type II sugar chain antibodies did not detect either G1 or G2 (data not shown). Fig. 6 displays the mass spectrum obtained by direct analysis of G2 upon purification. The molecular ion peaks shown in the lower part of Fig. 6 indicated that the structure was Cer-Hex-Hex-Hex-HexNAc (being Cer, ceramide; Hex, hexose; and HexNAc, N-acetylhexoseamine). These results suggested that the sugar chains of the G1 and $\mathrm{G} 2$ bands were both similar to the sugar chain of $\mathrm{Gb}_{4} \mathrm{Cer}$. As shown in Fig. 5, the fatty acid composition exhibited no appreciable differences between G1 and G2. Analysis of the composition of the long-chain base by TLC (Fig. 4B) revealed that it was composed of sphingosine, dihydrosphingosine and phytosphingosine in G1, while it contained sphingosine and dihydrosphingosine in G2. Thus, the presence of phytosphingosine was characteristic of G1. Based on these results, the ceramide in the G1 band that was only detected in well-differentiated endometrial cancer was observed to contain phytosphingosine.

Collectively, fatty acid and sphingosine in ceramides from specific neutral glycolipids from well-differentiated adenocarcinomas were revealed to be hydroxylated.

\section{Discussion}

In the present study, it was observed that glycolipids with a sugar chain longer than $\mathrm{Gb}_{4}$ Cer tended to be present in well-differentiated endometrial cancer (Fig. 1). In our previous study (3), such a longer chain glycolipid was not detected in the normal endometrium. Dihydrosphingosine was also detected as a long-chain base constituent of ceramide in endometrial cancer, while it was not detected previously in the normal endometrium (3). These differences between the normal endometrium and endometrial cancer suggest that the processes of ceramide and sugar chain synthesis differ between normal endometrial cells and endometrial cancer cells. Further studies are warranted to investigate glycolipids exhibiting slower migration than $\mathrm{Gb}_{4} \mathrm{Cer}$ on TLC in well-differentiated endometrial cancer, since there was considerable individual variation. Analysis by immunostaining may be necessary for this purpose, as the migration rate on TLC suggests that blood group substances may be present. 
The current study also investigated whether there were differences in the composition of neutral glycolipids regarding the degree of differentiation of endometrial cancer. The $\mathrm{Gb}_{3} \mathrm{Cer}$ band with a lower migration rate compared with the upper band of $\mathrm{Gb}_{3} \mathrm{Cer}$ on TLC had two components in well-differentiated cancer, while it had only one component in poorly differentiated cancer. This difference was associated with the composition of the fatty acids forming the ceramide. Namely, when the number of carbon atoms was $\geq 20$, non-hydroxy fatty acids were increased in well-differentiated cancer, while $\alpha$-hydroxy fatty acids were increased in poorly differentiated cancer. $\mathrm{Gb}_{4} \mathrm{Cer}$ also had two components in well-differentiated cancer vs. one component in poorly differentiated cancer. $\mathrm{Gb}_{4}$ Cer containing phytosphingosine was specifically identified in well-differentiated cancer.

Although it is unclear why such changes in glycolipids with a sugar chain longer than $\mathrm{Gb}_{4} \mathrm{Cer}$ and hydroxylated ceramides occur due to tumor differentiation, the following mechanisms could be proposed: First, it has been previously reported that the substrate specificity of the glycolipid-synthesizing glycosyltransferase influences the structure of ceramide (14); thus, glycosyltransferase may change with the extent of tumor differentiation, and this may cause the aforementioned band to occur specifically in well-differentiated cancer. Second, $\mathrm{Gb}_{3} \mathrm{Cer}$ and $\mathrm{Gb}_{4}$ Cer both exhibited two bands in well-differentiated cancer (Fig. 2A and B), suggesting that certain change may occur prior to ceramide synthesis that leads to differences in the composition of fatty acids and the long-chain base employed for ceramide synthesis between well- and poorly differentiated cancer. Although the meaning of these changes is unclear, it is possible to suggest that the amount of hydrophobic ceramide inserted into the cell membrane varies with the extent of tumor differentiation, resulting in differences on cell membrane structure between well- and poorly differentiated cancer that may be involved in determining the degree of malignancy of endometrial cancer. Long-chain bases have been reported to regulate the behavior of cancer (15). Therefore, it is necessary to further analyze such changes in ceramide regarding tumor differentiation at the level of ceramide synthesis.

In conclusion, the present study analyzed neutral glycolipids in endometrial cancer and revealed novel findings concerning ceramide. The results of the current study suggested that a number of the biological characteristics of the normal endometrium and endometrial cancer could be associated with the properties of these glycolipids and their ceramide structures.

\section{Acknowledgements}

The present study was partly supported by a grant-in-aid for scientific research from the Japanese Ministry of Education,
Culture, Sports, Science and Technology (MEXT; Tokyo, Japan; grant no. 26462538), the MEXT Supported Program for the Strategic Research Foundation at Private Universities, 2012-2014 (grant no. S1201001) and a grant from Tokai University Research Aid (Isehara, Japan; grant no. 2014012).

\section{References}

1. Iwamori M: A new turning point in glycosphingolipid Research. Hum Cell 18: 117-133, 2005.

2. Kubushiro K, Kojima K, Mikami M, Nozawa S, Iizuka R, Iwamori $M$ and Nagai Y: Menstrual cycle-associated alteration of sulfogalactosylceramide in human uterine endometrium: Possible induction of glycolipid sulfation by sex steroid hormones. Arch Biochem Biophys 268: 129-123, 1989.

3. Mikami M, Tukazaki K, Nozawa S, Iwamori M and Nagai Y: Menstrual cycle-associated expression of 2-hydroxy fatty acyl phytosphingosine-containing GlcCer, LacCer and Gb3Cer in human uterine endometrium. Biochim Biophys Acta 1125: 104-109, 1992.

4. Wegner MS, Wanger RA, Oertel S, Brachtendorf S, Hartmann D, Schiffmann S, Marschalek R, Schreiber Y, Ferreirós N, Geisslinger G and Grösch S: Ceramide synthases CerS4 and CerS5 are upregulated by $17 \beta$-estradiol and GPER1 via AP-1 in human breast cancer cells. Biochem Pharmacol 92: 577-589, 2014.

5. Merrill AH Jr: De novo sphingolipid biosynthesis: A necessary, but dangerous, pathway. J Biol Chem 277: 25843-25846, 2002.

6. Omae F, Miyazaki M, Enomoto A, Suzuki M, Suzuki Y and Suzuki A: DES2 protein is responsible for phytoceramide biosynthesis in the mouse small intestine. Biochem J 379: 687-695, 2004.

7. Fader AN, Arriba LN, Frasure HE and von Gruenigen VE: Endometrial cancer and obesity: Epidemiology, biomarkers, prevention and survivorship. Gynecol Oncol 114: 121-127, 2009.

8. Soper JT, McCarty KS Jr, Hinshaw W, Creasman WT, McCarty KS Sr and Clarke-Pearson DL: Cytoplasmic estrogen and progesterone receptor content of uterine sarcomas. Am J Obstet Gynecol 150: 342-348, 1984.

9. Sherman ME: Theories of endometrial carcinogenesis: A multidisciplinary approach. Mod Pathol 13: 295-308, 2000.

10. Montalto SA, Hakmi A, Moth P, Raju KS, Coutts M, Papadopoulos AJ and Devaja O: Well differentiated endometrioid adenocarcinoma of the uterus: A cancer unit or centre case? Eur J Gynaecol Oncol 30: 35-39, 2009.

11. Kiguchi K, Iwamori Y, Suzuki N, Kobayashi Y, Ishizuka B, Ishiwata I, Kita T, Kikuchi Y and Iwamori M: Characteristic expression of globotriaosyl ceramide in human ovarian carcinoma-derived cells with anticancer drug resistance. Cancer Sci 97: 1321-610, 2006.

12. Takehara K, Kubushiro K, Kiguchi K, Ishiwata I, Tsukazaki K, Nozawa $\mathrm{S}$ and Iwamori M: Expression of glycolipids bearing Lewis phenotypes in tissues and cultured cells of human gynecological cancers. Jpn J Cancer Res 93: 1129-1137, 2002.

13. Kiguchi K, Takamatsu K, Tanaka J, Nozawa S, Iwamori M and Nagai Y: Glycosphingolipids of various human ovarian tumors: A significantly high expression of I3SO3GalCer and Lewis antigen in mucinous cystadenocarcinoma. Cancer Res 52: 416-421, 1992.

14. Kartal Yandım M, Apohan E and Baran Y: Therapeutic potential of targeting ceramide/glucosylceramide pathway in cancer. Cancer Chemother Pharmacol 71: 13-20, 2013.

15. Liu J, Beckman BS and Foroozesh M: A review of ceramide analogs as potential anticancer agents. Future Med Chem 5: 1405-1421, 2013. 